\title{
Hamiltonian form for massless higher-spin fermions
}

\author{
C. Aragone* and S. Deser \\ Physics Department, Brandeis University, Waltham, Massachusetts 02254
}

(Received 2 May 1979)

\begin{abstract}
The action of the free massless spin-5/2 tensor-spinor field $\psi_{\mu \nu}$ is decomposed into its canonical form. It is shown to depend on a single gauge-invariant helicity $\pm 5 / 2$ spinor, the transverse-traceless and $\gamma$-transverse part of the spatial components $\psi_{i j}$, which obeys the ordinary Dirac equation. The remaining nine components are explicitly removed using invariance of the action under three local gauge functions, to each of which is associated a Lagrange multiplier, constraint variable, and gauge component. The analysis of the higher-half-integer-spin cases is then shown to follow in a uniform way, in terms of suitably defined field variables.
\end{abstract}

\section{INTRODUCTION}

Since the advent of supergravity ${ }^{1}$ there has been interest in the massless spin- $\frac{5}{2}$ field as a possible alternative fermionic companion to the graviton. Although there are grave a priori difficulties in achieving a consistent theory of helicity- $-\frac{5}{2}$-gravity coupling, ${ }^{2,8}$ the field is worth investigating in its own right as an example of higher-half-integralhelicity systems. The purpose of this work is to derive the canonical properties of higher-spin fermions, with emphasis on massless spin- $-\frac{5}{2}$ theory. First, we shall show for spin $\frac{5}{2}$ how its fundamental dynamical variables reduce from the original 10 spinor components $\psi_{\mu \nu}$ of a symmetric tensor-spinor to a single spinor, which suffices to describe the pure helicity $\pm \frac{5}{2}$ content and obeys the ordinary Dirac equation. We shall then examine arbitrary half-integral spins and show that their analysis follows in the same way when suitable field variables are chosen.

Our starting point for spin $\frac{5}{2}$ will be the action first given by Schwinger, ${ }^{3}$ together with the three local gauge invariances implicit in his Eq.

(3-3.114). For higher spins, we follow Fang and Fronsdal's ${ }^{4}$ extension of Schwinger's spin- $\frac{5}{2}$ action where the gauge-invariance properties of the basic symmetric variables are manifest. For the spin$\frac{5}{2}$ field $\psi_{\mu \nu}$, the reduction from 10 components to 1 component is easily understood given the number (3) of local gauge invariances: Each gauge invariance, for a first-order fermionic system, removes three components, one being a Lagrange multiplier, the second the associated constraint variable, and the third a pure gauge. Therefore, 3 local gauges are just the correct number to reduce the $10 \psi_{\mu_{\nu}}$ to a single one (as against 4 gauges required for the $h_{\mu \nu}$ tensor in the secondorder spin-2 massless theory). We shall show that the remaining variable is, as might be expected, related to the "TT" transverse-traceless part of the spatial components $\psi_{i j}$; since there are two of these, the final restriction is $\gamma$ trans- versality: $\gamma_{i} \psi_{i j}^{\mathrm{TTt}} \equiv 0$. This single spinor obeys the Dirac equation.

\section{THE SPIN-5/2 ACTION}

The action given in Ref. 3 reads (we use real rather than imaginary $\gamma$ matrices for our Majorana system, $\frac{1}{2}\left\{\gamma_{\mu}, \gamma_{\nu}\right\}=\eta_{\mu \nu}, \eta_{00}=-1, \gamma_{5}$ is real, $\gamma_{5}^{2}$ $=-1$, and $\left.\gamma_{\nu \alpha \lambda} \equiv \epsilon_{\nu \alpha \lambda_{k}} \gamma^{k} \gamma_{5}\right)$

$$
\begin{aligned}
I(\psi) \equiv-\frac{i}{2} \int\left(d^{4} x\right) & \left(\bar{\psi}^{\mu \nu} \not \partial \psi_{\mu \nu}+\frac{1}{2} \bar{\psi} \not \partial \psi\right. \\
& \left.-2 \bar{\psi} \partial_{\nu} \psi_{\nu}-2 \bar{\psi} \bar{\psi}_{\mu \nu} \gamma^{\nu \alpha \lambda} \partial_{\alpha} \psi_{\mu \lambda}\right),
\end{aligned}
$$

where $\psi \equiv \psi_{\alpha}^{\alpha}, \psi_{\mu} \equiv \gamma_{\alpha} \psi_{\mu}^{\alpha}$. [Three recent discussions ${ }^{4,5,6}$ use slightly different actions, but these are equivalent to (1).]

It is invariant under the transformations generated by the $\gamma$-traceless vector-spinor $\tilde{\zeta}_{\mu}$ :

$$
\delta \psi_{\mu \nu}=\tilde{\zeta}_{\mu, \nu}+\tilde{\zeta}_{\nu, \mu}, \quad \gamma^{\alpha} \tilde{\zeta}_{\alpha} \equiv 0,
$$

as is implicit in Eqs. (3-3.114) of Ref. 3, which states that any source $\tau^{\mu \nu}$ of the field equations (with coupling $\int \bar{\psi}_{\mu \nu} \tau^{\mu \nu}$ ) is subject to the condition $\partial_{\mu}\left(\tau^{\mu \nu}-\frac{1}{4} \gamma^{\nu} \gamma_{\alpha} \tau^{\mu \alpha}\right) \equiv \partial_{\mu} \tau^{\mu \tilde{\nu}}=0, \gamma_{\nu} \tau^{\mu \tilde{\nu}}=0$.

We begin with a very compact intuitive reduction. Its purpose is to explain, using dynamical arguments, how the constraints first given in Ref. 5 for spin $\frac{5}{2}$ arise, and to clarify their content. This will also motivate the generalization to higher spin in the last section. To each gauge freedom there is associated a Lagrange multiplier, a constraint equation (which eliminates one variable), and a gauge variable, one which may be set to zero by a convenient choice of gauge. The simplest way to find the three Lagrange multipliers is to find those $\psi_{\mu \nu}$ components which vary under (2) in a way involving a time derivative. [For spin $\frac{3}{2}$, where the field is a vector-spinor $\psi_{\mu}$, with invariance under $\delta \psi_{\mu} \equiv \partial_{\mu} \alpha(x), \psi_{0}$ is the multiplier, $\sigma^{i j} \partial_{i} \psi_{j}=0$ the constraint, and the longitudinal component $\psi_{, j}^{L}$ or the $\gamma$ longitudinal one $\gamma_{i} \psi_{i}$ are examples of a gauge variable.] In our case, the 
multipliers are three of the four $\psi_{0 \mu}$, since

$$
\chi \equiv \gamma_{0} \gamma^{i} \psi_{i 0}-\frac{1}{2} \psi_{00} \equiv \gamma_{0} \gamma^{\mu} \psi_{\mu 0}+\frac{1}{2} \psi_{00},
$$

does not vary into a time derivative. (We shall see in the next section that this argument stems from the Bianchi identities, which always ensure consistency of the constraints: If these are satisfied at a certain instant, they are automatically satisfied at all times by virtue of the time evolution equations.)

The corresponding three constraints are $\left(\psi_{i}\right.$ $\left.\equiv \psi_{i j, j}, \eta_{i} \equiv \gamma_{j} \psi_{i j}\right)$

$$
C_{i} \equiv \psi_{i}-\not \varnothing \eta_{i}-\frac{1}{2}\left(\psi_{k k}+2 \chi\right)_{i}=0 \text {. }
$$

Finally, the gauge transformations on the seven components $\left(\psi_{i j}, \chi\right)$ are

$$
\delta \psi_{i j}=\tilde{\zeta}_{i, j}+\tilde{\zeta}_{j, i}, \quad \delta \chi=\not x \gamma^{0} \zeta_{0} .
$$

We may use these to eliminate $\chi$ and two among the three longitudinal components $\phi_{i, j}+\phi_{j, i}$ of $\psi_{i j}$ (since $\gamma^{i} \widetilde{\zeta}_{i}=-\gamma^{0} \tilde{\zeta}_{0}$ constrains the $\tilde{\zeta}_{i}, \gamma^{i} \psi_{i}$ cannot be removed once we have taken $\tilde{\zeta}_{0}$ such that $\chi=0$ ), or else eliminate all three $\phi_{i}$.

With the first choice, $\phi_{i}$ will have the form $\gamma_{i} \lambda$ in the general decomposition

$$
\psi_{i j}=\psi_{i j}^{\mathrm{TT}}+\psi_{i j}^{\mathrm{T}}+\left(\phi_{i, j}+\phi_{j, i}\right),
$$

where

$$
\psi_{i j, j}^{\mathrm{TT}}=\psi_{i i}^{\mathrm{TT}} \equiv 0 \equiv \psi_{i j, j}^{\mathrm{T}} .
$$

In either gauge, use of the $\gamma$ trace and divergence of (4) immediately shows that only $\psi_{i j}^{\mathrm{TT}}$ survives and therefore simply satisfies

$$
\not \gamma_{k} \psi_{i k}^{\mathrm{TT}}=0 \rightarrow \gamma_{k} \psi_{i k}^{\mathrm{TT}}=0 \text {. }
$$

At first sight, these seem to be three further conditions on the two components of $\psi_{i j}^{\mathrm{TT}}$. However, there is only one condition there; as may easily be seen by noting that this $\gamma$-transverse $\psi_{i j}^{\text {TTt }}$ satisfies only 5 rather than " 7 " conditions, since those are related by the two identities $\partial_{i}\left(\gamma_{j} \psi_{i j}^{\mathrm{TTt}}\right) \equiv 0$ and $\gamma_{j}\left(\partial_{i} \psi_{i j}^{\mathrm{TTt}}\right) \equiv 0$. This is also clear algebraically by defining the transverse direction at any point to be $x$; then $\psi_{i j}^{\mathrm{TT}}$ has the form

$$
\psi_{i j}^{\mathrm{TT}} \equiv\left(\begin{array}{ccc}
0 & 0 & 0 \\
0 & \alpha & \beta \\
0 & \beta & -\alpha
\end{array}\right),
$$

and $\gamma_{j} \psi_{i j}^{\mathrm{TTt}}=0$ are just the two conditions $\gamma_{2} \alpha$ $+\gamma_{3} \beta=0$ and $\gamma_{2} \beta-\gamma_{3} \alpha=0$ which are equivalent. We shall be more explicit about the projection operators for $\psi^{\text {T Tt }}$ below. In our gauge, then, $\psi_{\mu \nu} \equiv\left(\psi_{i j}^{\mathrm{TT}}, 0\right)$ and the action (1) immediately reads

$$
I=-\frac{i}{2} \int \bar{\psi}_{i j}^{\mathrm{TTt}} \not \partial \psi_{i j}^{\mathrm{TTt}}\left(d^{4} x\right),
$$

as required for a pure helicity $\pm \frac{5}{2}$ particle.

\section{BIANCHI IDENTITIES, CONSTRAINTS, AND LAGRANGE MULTIPLIERS}

The action (1) gives

$$
\begin{aligned}
\delta I & =\int\left(d^{4} x\right) \delta \bar{\psi}^{\mu \nu} \hat{S}_{\mu \nu} \\
& =-\int\left(d^{4} x\right) \delta \bar{\psi}^{\mu \nu}\left(\sigma_{\mu \alpha} S_{\nu}^{\alpha}+\sigma_{\nu \alpha} S_{\mu}^{\alpha}+\frac{1}{2} \eta_{\mu \nu} S_{\alpha}^{\alpha}\right),
\end{aligned}
$$

where $\hat{S_{\mu \nu}}=0$ is Schwinger's form of the massless spin- $\frac{5}{2}$ field equations,

$$
\begin{aligned}
\hat{S}_{\mu \nu}(\psi) & \equiv \not \partial \psi_{\mu \nu}+2^{-1} \eta_{\mu \nu} \not \partial \psi-\eta_{\mu \nu} \partial_{\alpha} \not \phi^{\alpha}-2^{-1}\left(\gamma_{\mu} \partial_{\nu}+\gamma_{\nu} \partial_{\mu}\right) \psi \\
& -\gamma_{\mu \alpha \sigma} \partial^{\alpha} \psi_{\nu}^{\sigma}-\gamma_{\nu \alpha \sigma} \partial^{\alpha} \psi_{\mu}^{\sigma}=0,
\end{aligned}
$$

while

$S_{\mu \nu} \equiv \gamma^{\alpha} \Omega_{\mu \nu, \alpha} \equiv \gamma^{\alpha} 2^{-1}\left(\psi_{\nu \alpha, \mu}+\psi_{\mu \alpha, \nu}-\psi_{\mu \nu, \alpha}\right)=0$,

is the simpler (and equivalent) version. ${ }^{5}$

Invariance of the action under the local gauge transformations (2) implies the existence of three independent Bianchi identities:

$\delta_{\zeta} I=\int\left(d^{4} x\right) \bar{\zeta}^{\tilde{\mu}}\left(\partial_{\nu} S_{\mu}^{\nu}-2^{-1} \partial_{\mu} S_{\alpha}^{\alpha}-2^{-1} \not \partial S_{\mu}\right) \equiv 0$,

$I_{i} \equiv \partial_{0}\left(S_{i}^{0}-2^{-1} \gamma^{0} \phi_{i}\right)+\partial_{j}\left(S_{i}^{j}-2^{-1} \delta_{i}^{j} S-2^{-1} \gamma^{j} \phi_{i}\right) \equiv 0$,

where $\phi_{\mu} \equiv \gamma^{\alpha} S_{\mu \alpha}$. From this last equation, one might guess that the constraints $C_{i}$ are closely related to $S_{i}^{0}-2^{-1} \gamma^{0} \phi_{i}$; indeed, it turns out that

$$
\hat{S}_{i}^{0}+4^{-1} \gamma_{i} \gamma^{\alpha} \hat{S}_{\alpha}^{0} \equiv S_{i}^{0}-2^{-1} \gamma^{0} \not \phi_{i} \equiv \gamma^{0} C_{i} \text {. }
$$

We also observe from Eq. (4) that the $C_{i}$ are true constraints in that they contain no time derivatives, nor the variables $\psi_{0 i}$. Equation (12b) then ensures that the constraints are preserved under time evolution, since $C_{i}$ is proportional to the remaining field equations at every instant.

Comparing the first and last members of Eq. (13), it is clear that the Lagrange multipliers $n_{i}$ associated with the constraints $C_{i}$ are

$$
n_{i} \equiv \psi_{0 i}+\gamma_{i} \gamma_{j} \psi_{0 j},
$$

as we shall explicitly exhibit in the next section.

\section{REDUCTION TO THE CANONICAL ACTION AND QUANTIZATION}

In terms of $\psi_{i j}, \eta_{i}, \psi_{i}, n_{i}, \chi,{ }_{3} \psi \equiv \psi_{i i}$ and their transposes, the action (1) reads

$$
\begin{aligned}
I=i^{-1} \int\left(d^{4} x\right) & {\left[2^{-2}{ }_{3} \psi_{3} \dot{\psi}-\chi \dot{\chi}+{ }_{3} \psi \dot{\chi}-2^{-1} \psi_{i j} \dot{\psi}_{i j}+\eta_{i} \dot{\eta}_{i}\right.} \\
& +2^{-1} \psi_{i j} \gamma^{0} \not \psi_{i j}-\eta_{i} \gamma^{0} \not \eta_{i}+2 \psi_{i} \gamma^{0} \eta_{i} \\
& -2 \chi \gamma^{0} \not \supset \chi-\left(\chi-2^{-1}{ }_{3} \psi\right) \gamma^{0} \not x\left(\chi-2^{-1}{ }_{3} \psi\right) \\
& \left.\left.+{ }_{3} \psi-2 \chi\right) \gamma^{0} \gamma_{i} \psi_{i}+2 n_{i} C_{i}\right],
\end{aligned}
$$


where the constraint and Lagrange multiplier structure is evident in the last term.

The next step in the reduction process is to solve the differential constraints. In order to do this, it is convenient to decompose the symmetric spatial tensor-spinor $\psi_{i j}$ in the form $\left[\rho_{i} \equiv(-\Delta)^{-1 / 2} \partial_{i}\right.$, $\left.\kappa_{i} \equiv p_{i j} \gamma_{j} \equiv\left(\delta_{i j}+\rho_{i} \rho_{j}\right) \gamma_{j}\right]$

$$
\begin{aligned}
\psi_{i j}= & \psi_{i j}^{\mathrm{TTt}}+\psi_{i j}^{\mathrm{TT}} l+\psi_{i j}^{T}+\rho_{i} \psi_{2 j}^{T t}+\rho_{j} \psi_{2 i}^{T t} \\
& +\left(\rho_{i} \kappa_{j}+\rho_{j} \kappa_{i}\right) \psi^{T l}+\rho_{i} \rho_{j} \psi^{L}
\end{aligned}
$$

where $\psi_{i j}^{\mathrm{TTt}}$ is the pure helicity- $\frac{5}{2}$ part of $\psi_{i j}$, $\psi_{i j}^{\mathrm{TT} l}$ contains one helicity- $\frac{3}{2}$ vector-spinor, $\psi_{i j}^{T}$ $=p_{i j} \psi^{T}$ contains the usual scalar-spinor, $\psi_{2 i}^{T t}$ is the second helicity- $\frac{3}{2}$ vector-spinor, and $\psi^{T l}$ and $\psi^{L}$ are pure $\frac{1}{2}$ spinors: Eq. (16) decomposes $\psi_{i j}$ into its 6 intrinsic helicity components. Details of this decomposition are given in Appendix A.

This type of projection has previously been used in Ref. 7 to decompose a spatial vector-spinor $\tilde{\zeta}_{i}$ into its 3 pure helicity parts:

$$
\tilde{\zeta}_{i}=\zeta_{i}^{T t}+\kappa_{i} \zeta^{T l}+\rho_{i} \zeta^{L} .
$$

In that case, $\zeta_{i}^{T t}$ is a pure helicity- $-\frac{3}{2}$ field, and both $\zeta^{T l}$ and $\zeta^{L}$ have helicity $\frac{1}{2}$.

Making use of the intrinsic decomposition (16), the constraints $C_{i}=0$ can be solved in a gaugeindependent way. Their solution is

$$
\begin{aligned}
& \psi^{T}=0=\psi_{1 i}^{T t}, \\
& 2 \chi=\psi^{L}-4 \phi \psi^{T l} .
\end{aligned}
$$

Physically, (18) states that the TT part of $\psi_{i j}$ only contains a pure helicity- $\frac{5}{2}$ part $\psi_{i j}^{T T t}$ and that $\chi$ is not independent, but is a linear combination of two helicity $-\frac{1}{2}$ parts of $\psi_{i j}$.

Now we make use of the gauge variance of some components of $\psi_{i j}$. Under a gauge transformation like (5), where $\tilde{\zeta}_{i}$ has been projected as in Eq. (17), the only 3 components of $\psi_{i j}$ which undergo a variation are the constituents of its "longitudinal" part, i. e.,

$$
\delta \psi_{2 i}^{T t}=\zeta_{i}^{T t}, \quad \delta \psi^{T l}=\zeta^{T l}, \quad \delta \psi^{L}=2 \zeta^{L}
$$

That means that the action (15) cannot depend upon $\left(\psi_{2 i}^{T t}, \psi^{T l}, \psi^{L}\right)$ and therefore that one can conveniently compute its value by making a suitable gauge choice. We shall use the gauge $\psi^{I}=\psi^{T t}$ $=\psi_{2 i}^{T t}=0$; Eqs. (18) then imply that $\chi=0$ and the action (15) reaches the final unconstrained and gauge-independent form (9) [we have also used the vanishing of $\eta_{i} \equiv \psi_{1}^{T t}+\kappa_{i} \psi^{T}$ implied by Eq. (18)].

The TTt projection also enables us to obtain the canonical anticommutation relations in terms of the basic variable

$$
\psi_{i j}^{\mathrm{TTt}}=P_{i j k l} Q_{k l m n} P_{m n p q} \psi_{p q} \equiv \pi_{i j}^{p q} \psi_{p q},
$$

where

$$
\begin{aligned}
P_{i j k l} & \equiv p_{i k} p_{j l}-2^{-1} p_{i j} p_{k l}, \\
Q_{k l m n} & \equiv 2^{-2}\left(\delta_{k m} \delta_{l n}+\delta_{k n} \delta_{l m}+\gamma_{k} \gamma_{m} \delta_{l n}+\gamma_{l} \gamma_{m} \delta_{k n}\right)
\end{aligned}
$$

We find

$$
\begin{aligned}
\left\{*^{*} \psi_{i j L}^{\mathrm{TTt}}\left(\overrightarrow{\mathrm{r}}_{1}\right), \psi_{k l L}^{\mathrm{T} \mathrm{Tt}}(0)\right\}= & { }_{1} \pi_{i j}{ }^{i^{\prime} j^{\prime}}{ }_{2} \pi_{k l}{ }^{k^{\prime} l^{\prime}} \\
& \times \frac{1}{2}\left(\delta_{i k} \delta_{j l}+\delta_{i l} \delta_{j k}\right) P_{L} \delta^{3}\left(\overrightarrow{\mathrm{r}}_{1}\right),
\end{aligned}
$$

where (as usual for Majorana spinors) the $\psi, * \psi$ must be taken as, for instance, the left (or right) components: $\psi_{L}, * \psi_{L}=2^{-1}\left(1 \pm i \gamma_{5}\right) \psi \equiv P_{L, R} \psi$.

\section{ARBITRARY HELICITY}

Having analyzed helicity $\frac{5}{2}$ (which is closely analogous to the helicity $-\frac{3}{2}$ reduction ${ }^{7}$ ), we now show that higher helicities also follow the same pattern. If the general $s+\frac{1}{2}$ field is characterized by a totally symmetric world tensor-spinor $\psi_{\mu_{1}} \ldots \mu_{s}$, it has $N_{4}(s) \equiv 6^{-1}(s+1)(s+2)(s+3)$ components. Assuming the associated gauge invariance to have the form (2), with symmetric $\tilde{\zeta}_{\mu_{2} \cdots \mu}$, reduces this by $3\left[N_{4}(s-1)-N_{4}(s-2)\right]$, taking the $\gamma$ tracelessness of $\tilde{\zeta}$ into account. However, this still leaves $N_{4}(s-3)+1$ components, so that for $s>2$ a further restriction is needed. This is provided by the requirement (first noted empirically in Ref. 4) that

$$
\psi^{\prime \prime \prime} \equiv \gamma^{\lambda} \gamma^{\mu} \gamma^{\nu} \psi_{\lambda \mu \nu \mu_{4} \cdots \mu_{s}} \equiv \gamma^{\lambda} \psi_{\alpha \alpha \lambda \mu_{4} \ldots \mu_{s}}=0,
$$

which are precisely $N_{4}(s-3)$ conditions.

Now, it is of course not permitted just to set a component such as $\psi^{\prime \prime \prime}=0$ by hand in the action (and, a fortiori, in the field equations obtained by varying all the $\psi$ components independently), particularly for gauge fields. We shall therefore show that this can be accomplished here, because $\psi^{\prime \prime \prime}$ is gauge invariant; setting it to zero is then a condition preserved under choice of gauge. (This automatically implies that all higher traces, $\psi^{\prime \prime \prime \prime}$, etc., are also invariants and need not be treated separately, vanishing when $\psi^{\prime \prime \prime}$ does.)

We shall also use this invariance to obtain the action's dependence on $\psi^{\prime \prime \prime}$. Actually, $\psi^{\prime \prime \prime}$ is the lowest contraction to be invariant, since

$$
\begin{aligned}
& \delta \psi_{\mu_{2} \cdots \mu_{s}}^{\prime}=s^{-1} \not \widetilde{\zeta}_{\mu_{2} \cdots \mu_{s}} \neq 0, \\
& \delta \psi_{\mu_{3} \cdots \mu_{s}}^{\prime \prime}=2 s^{-1}(\partial \cdot \tilde{\zeta})_{\mu_{3} \cdots \mu_{s}} \neq 0 .
\end{aligned}
$$

From these, it follows that

$$
\delta \psi_{\mu_{4}}^{\prime \prime \prime} \cdots \mu_{s} \equiv 2 s^{-1} \gamma^{\alpha} \cdot(\partial \cdot \tilde{\zeta})_{\alpha \mu_{4} \cdots \mu_{s}} \equiv 0 .
$$

[In particular, then, neither $\psi^{\prime}$ nor $\psi^{\prime \prime}$ can be meaningfully restricted, because of (23); indeed, 
$\psi_{\mu_{2}, \ldots \mu_{s}}^{\prime}=0$ is a useful gauge choice.] Consider now dependence of the action on $\psi^{\prime \prime \prime}$; we show that this is always of the form $\bar{\psi}^{\prime \prime \prime} \Gamma \partial \psi^{\prime \prime \prime}$ so that, if initially present, it can be invariantly removed, leaving dependence only on the remaining components $\psi^{*}$ out of which $\psi^{\prime \prime \prime}$ dependence has been projected, i.e., $I(\psi) \equiv I\left(\psi^{*}\right)+I\left(\psi^{\prime \prime \prime}\right)$. The total $\psi^{\prime \prime \prime}$ dependence is a sum of the form $\psi^{\prime \prime \prime}(\Sigma \Gamma \partial \psi)$. Since there are no other terms involving $\psi^{\prime \prime \prime}$, by construction, the coefficient of $\psi^{\prime \prime \prime}$ must be gauge invariant by itself. But there are no such invariants available, constructed out of $\psi^{*}$, as is easily checked using (23) and index counting (essentially only $\bar{\psi}^{\prime \prime \prime} \partial \cdot \psi^{\prime \prime}$ is possible, and it is not invariant). The remaining possibility then is $\sim \bar{\psi}^{\prime \prime} \Gamma \partial \psi^{\prime \prime \prime}$, which, for example, when $s=\frac{7}{2}$, is a pure spin $-\frac{1}{2}$ object $\sim \bar{\lambda} \phi_{\lambda}$ while for $s=\frac{9}{2}$, it could include pure spin $\frac{3}{2}$ or a mixture of $\frac{3}{2}$ and $\frac{1}{2}$, etc. (Of course, there is no relation be- tween the invariances of such unwanted spins under $\delta \psi_{\mu}^{\prime \prime \prime}=\theta_{\mu} \alpha$ for $\frac{3}{2}$ and the $\tilde{\xi}$ invariance of the original spin- $\frac{9}{2}$ system; indeed, no scalar $\alpha$ can be constructed from $\tilde{\xi}_{\mu \nu \alpha}$. ) In any case, if one's initial action were gauge invariant and contained such bilinears in $\psi^{\prime \prime \prime}$, one could simply add appropriate terms to ensure pure $s+\frac{1}{2}$ content (the projection to $\psi^{*}$ is a local algebraic one and does not introduce new poles). Equivalently, one may simply require the $\psi$ to be of the $\psi^{*}$ type (just as they are taken to be totally symmetric) since there is consistency with the postulated gauge invariance. In the reduced action, the $\psi^{*}$ are freely varied and yield field equations which reflect this projection in the vanishing of their triple $\gamma$ trace (see Appendix B). This argument provides the legitimization of the procedure followed in Refs. 4 and 6 where the action is taken to be

$$
\begin{aligned}
I_{s+1 / 2}=-\frac{i}{2} \int\left(d^{4} x\right)\left[\bar{\psi}_{\mu_{1} \cdots \mu_{s}} \phi \psi_{\mu_{1} \cdots \mu_{s}}+\frac{1}{2}(s-1)^{2} \bar{\psi}_{\mu_{3} \cdots \mu_{s}}^{n} \not \partial \psi_{\mu_{3} \cdots \mu_{s}}^{\prime \prime}\right. \\
\left.-s \bar{\psi}_{\mu_{1} \cdots \mu_{s-1} \nu} \gamma^{\nu \alpha \lambda} \partial_{\alpha} \psi_{\mu_{1} \cdots \mu_{s-1} \lambda}-s(s-1) \bar{\psi}_{\mu_{3} \cdots \mu_{s}}^{\prime \prime} \theta_{\mu} \gamma_{\nu} \psi_{\mu \nu} \cdots \mu_{s}\right],
\end{aligned}
$$

whose field equations do in fact explicitly contain $\psi^{\prime \prime \prime}$ as they stand, and $\psi^{\prime \prime \prime}$ is set to zero by hand.

Thus, removal from the general reducible $\psi$ of its gauge-invariant $\psi^{\prime \prime \prime}$ part completes the partial reduction brought about by gauge invariance itself, since the latter's function is to remove other irreducible components of $\psi$. Any quantity of the form $\tilde{\zeta}$ (which includes also $\psi^{\prime \prime}$ when $\psi^{\prime \prime \prime}=0$ ) is an irreducible representation of type $(j, j+1) \otimes(j$ $+1, j$ ) by virtue of its $\gamma$ transversality. (In $2-$ spinor notation, $\tilde{\zeta}_{\mu_{2} \cdots \mu_{s}} \sim \zeta_{A_{2} \cdots A_{s_{1}} B_{2} \cdots \dot{B}_{S} \dot{B}_{s+1}}$ $\otimes \bar{\zeta}_{B_{2} \cdots B_{s} B_{s+1}, \dot{A}_{2} \cdots \dot{A}_{s}}$.

Let us now turn to the canonical variables and see that the natural gauge-invariant component to describe the pure helicity $\pm\left(s+\frac{1}{2}\right)$ states remains the transverse-traceless and $\gamma$-transverse quantity $\psi_{i_{1} \cdots i_{s}}^{T} \cdots T_{s}{ }^{t}$, whose definition is as for spin $\frac{5}{2}$. Exactly the same arguments as before show that it has only one component for any $s$. For example, it is easy to see that $\psi_{i_{1}}^{T_{1}} \cdots_{i_{s}}^{T_{s}}$ has 2 components by counting the restrictions on $N_{3}(s) \equiv \frac{1}{2}(s+1)(s+2)$ due to transversality, $N_{3}(s-1)$, and tracelessness, $N_{3}(s-2)$ [not forgetting to subtract out, however, the $N_{3}(s-3)$ identities between the trace and transversality restrictions]. Is is then clear that

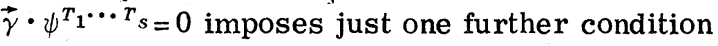
on the 2 components by either of the earlier arguments used for $s=2$. (It is also clear that setting the lower-spin gauge-invariant $\psi^{\prime \prime \prime}$ to zero

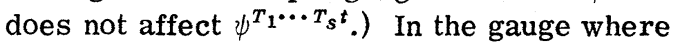

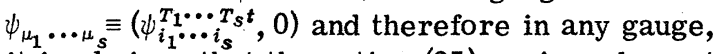
it is obvious that the action (25) again reduces to the (gauge-invariant) form

$$
I_{s+1 / 2}=-\frac{i}{2} \int \bar{\psi}_{i_{1}{ }_{1} \cdots i_{s}}^{T} s^{t} \not \partial \psi_{i_{1} \cdots i_{s}}^{T} \cdots T_{s}{ }^{t}\left(d^{4} x\right),
$$

as all other terms involve $\vec{\gamma}$ or $\vec{\nabla}$ traced components of $\psi$. Strictly speaking, one should check that all other components of $\psi_{\mu_{1}} \ldots \mu_{s}$ are removed by the constraints, etc., but this is really guaranteed by the general framework; it can be done in principle, being mainly a notational problem.

The counting of degrees of freedom and the reduced canonical form for the arbitrary $\left(s+\frac{1}{2}\right)$ case, then, is precisely analogous to that of $\frac{5}{2}$, with the exception that condition (22) (or the equivalent restrictions of $\psi$ to its $\psi^{*}$ subspace) is necessary (and implementable) in order to remove the unwanted separate lower-spin actions.

The existence and intuitively satisfactory behavior of free higher-spin fermionic theories in terms of their Abelian gauge properties suggests a possible connection with higher global gradings. ${ }^{9,10}$ However, it is now known ${ }^{11,12}$ that such algebras (with constant parameters $\xi_{\tilde{\mu}_{1}} \ldots$ ) have no particle representations.

Finally, we mention that there also exists a different representation of $s>\frac{3}{2}$ fermions in terms of nonsymmetric, vierbein-like, tensor-spinors, ${ }^{13}$ e.g., $\psi_{\mu \tilde{a}}\left(\gamma^{a} \psi_{\mu \tilde{a}} \equiv 0\right)$ for spin $\frac{5}{2}$, with corresponding actions of a uniform Rarita-Schwinger type. In terms of these, the gravitational coupling problems are described in a systematic and transparent way. 


\section{ACKNOWLEDGMENTS}

C.A. was the recipient of a John Simon Guggenheim Memorial Fellowship. The work of C.A. was supported in part by CONICIT (Venezuela) Grant No. S1-0972. The work of S.D. was supported in part by NSF Grant No. PHY-78-09644.

\section{APPENDIX A}

We present here some useful properties of the algebra of projections on spatial vector and secondorder tensor-spinors.

The elements of this algebra are the Riesz operators $\rho_{i} \equiv(-\Delta)^{-1 / 2} \partial_{i}, \gamma_{i}, p_{i j} \equiv \delta_{i j}+\rho_{i} \rho_{j}$, and $\kappa_{i} \equiv p_{i j} \gamma_{j}$.

A vector-spinor $\varphi_{i}$ is projected into three orthogonal subspaces according to

$$
\varphi_{i}=\varphi_{i}^{T t}+\kappa_{i} \varphi^{T l}+\rho_{i} \varphi^{L},
$$

where

$$
\varphi^{L}=-\rho_{i} \varphi_{i}, \quad \varphi^{T l}=2^{-1} \kappa_{i} \varphi_{i} .
$$

Therefore,

$$
\varphi_{i}^{T t} \equiv \varphi_{i}-2^{-1} \kappa_{i} \kappa_{j} \varphi_{j}+\rho_{i} \rho_{j} \varphi_{j} \equiv\left(p_{i j}-2^{-1} \kappa_{i} \kappa_{j}\right) \varphi_{j} .
$$

The elementary projectors $\rho_{i}, p_{i j}, \kappa_{i}$ satisfy the following relationships:

$$
\begin{aligned}
& \rho_{i} \kappa_{i}=0, \kappa_{i} \kappa_{i}=2=\gamma_{i} \kappa_{i}=-2 \phi \phi, \\
& \left\{\kappa_{i}, \kappa_{j}\right\}=\left\{\gamma_{i}, \kappa_{j}\right\}=2 p_{i j}, \quad\left\{\phi, \kappa_{j}\right\}=0, \\
& \left\{\gamma^{0}, \kappa_{i}\right\}=0=\left[\phi, \rho_{i}\right] .
\end{aligned}
$$

The inner product of two spatial vector-spinors $\varphi_{i}, \psi_{j}$ exhibits the orthogonality between the 3 projections given in (A1):

$$
\begin{aligned}
\int \varphi_{i} \psi_{i}\left(d^{3} x\right)= & \int\left(d^{3} x\right) \varphi_{i}^{T t} \varphi_{i}^{T t} \\
& +2 \int\left(d^{3} x\right) \varphi^{T \imath} \psi^{T l}+\int\left(d^{3} x\right) \varphi^{L} \psi^{L} .
\end{aligned}
$$

For a spatial symmetric tensor-spinor $\psi_{i j}$ the corresponding decomposition is given by

$$
\begin{aligned}
\psi_{i j}= & \psi_{i j}^{T T t}+4^{-1}\left(\kappa_{i} \psi_{i j}^{T t}+\kappa_{j} \psi_{1 i}^{T t}\right)+p_{i j} \psi^{T} \\
& +\rho_{i} \psi_{2 j}^{T t}+\rho_{j} \psi_{2 i}^{T t}+\left(\rho_{i} \kappa_{j}+\rho_{j} \kappa_{i}\right) \psi^{T l}+\rho_{i} \rho_{j} \psi^{L} .
\end{aligned}
$$

The inner product of two spatial symmetric tensorspinors $\varphi, \psi$ has the value (omitting obvious indices)

$$
\begin{aligned}
\int \varphi \psi\left(d^{3} x\right) & =\int\left(d^{3} x\right) \varphi^{T T t} \psi^{\mathrm{T} T t}+2^{-1} \int \varphi_{1}^{T t} \psi_{1}^{T t}\left(d^{3} x\right)+2 \int\left(d^{3} x\right) \varphi^{T} \psi^{T} \\
& +2 \int \varphi_{2}^{T t} \psi_{2}^{T t}\left(d^{3} x\right)+4 \int\left(d^{3} x\right) \varphi^{T} \psi^{T t}+\int \varphi^{L} \psi^{L}\left(d^{3} x\right)
\end{aligned}
$$

The values of $\psi_{i i} \equiv{ }_{3} \psi, \eta_{i} \equiv \gamma_{j} \psi_{i j}$, and $\psi_{i} \equiv \rho_{j} \psi_{i j}$ in terms of the six components of $\psi_{i j}$ are

$$
\begin{aligned}
{ }_{3} \psi= & 2 \psi^{T}-\psi^{L}, \quad \psi_{i}=-\psi_{2 i}^{T t}-\kappa_{i} \psi^{T l}-\rho_{i} \psi^{L}, \\
\eta_{i}= & \left(\psi_{1 i}^{T t}+\phi \psi_{2 i}^{T t}\right)+\kappa_{i}\left(\psi^{T}-\not \psi^{T l}\right) \\
& +\rho_{i}\left(\phi \psi^{L}+2 \psi^{T l}\right) .
\end{aligned}
$$

These equations are useful in establishing the values of these variables in various gauges.

\section{APPENDIX B}

The action for a helicity $s+\frac{1}{2}$ fermion was given in Eq. (25), where $\psi_{\mu_{1}} \ldots \mu_{s}$ is triple traceless. This fact allows us to write it in the form [understanding the ( ) as an omitted summation on all the different possibilities]

$$
\begin{aligned}
& \psi_{\mu_{1}}^{*} \cdots \mu_{s} \\
& \left.\quad \equiv \psi_{\mu_{1} \cdots \mu_{s}}-\frac{1}{4} s^{-1}(s-1)^{-1} \eta_{\left(\mu_{1} \mu_{2}\right.} \gamma_{\left.\mu_{3}\right)} \psi_{\mu_{4}}^{\prime \prime \prime} \cdots \mu_{s}\right) \\
& \quad+\cdots \\
& \quad+\frac{1}{21} s^{-1}(s-1)^{-1} b_{s} \eta_{\left(\mu_{1} \mu_{2}\right.} \eta_{\mu_{3} \mu_{4}} \cdots \eta_{\left.\mu_{s-1} \mu_{s}\right)} \psi^{(s)},
\end{aligned}
$$

where due to the gauge invariance of $\psi^{\prime \prime \prime}$, both the triple traceless $\psi_{\mu_{1}}^{*} \ldots \mu_{s}$ and the arbitrary symmetric $\psi_{\mu_{1}} \ldots \mu_{s}$ have the same gauge transformation law $\delta \psi_{\mu_{1} \ldots \mu_{s}}=\partial_{\left(\mu_{1}\right.} \tilde{\xi}_{\left.\mu_{2} \cdots \mu_{s}\right)}$, and $\hbar_{\mu_{s-1} \mu_{s}}$ stands for $\eta_{\mu_{s-1} \mu_{s}}$ when $s$ is even and for $\gamma_{\mu_{s}}$ when $s$ is odd; the $b_{s}$ coefficients [as well as the $a_{s}$ in Eq. (B5)] are defined recursively and may be computea straigntforwardly.

If we wish to obtain the field equations, we perform independent variations of $\psi^{*}$ in the action (25):

$$
\delta I_{s+1 / 2}=-\frac{i}{2} \int d^{4} x \delta \bar{\psi}_{\mu_{1} \cdots \mu_{s}}^{*} \hat{S}_{\mu_{1} \ldots \mu_{s}}\left(\psi^{*}\right),
$$

from which one can state that the field equations are given by

$$
\begin{aligned}
\hat{S}_{\mu_{1} \cdots \mu_{s}}^{*} \equiv & \hat{S}_{\mu_{1} \cdots \mu_{s}}\left(\psi^{*}\right) \\
& -\frac{1}{4} s^{-1}(s-1)^{-1} \eta_{\left(\mu_{1} \mu_{2}\right.} \gamma_{\left.\mu_{3}\right)} \hat{S}_{\mu_{4} \cdots \mu_{s}}^{\prime \prime \prime}{ }^{\prime \prime}+\cdots \\
& +\frac{1}{4} s^{-1}(s-1)^{-1} b_{s} \eta_{\left(\mu_{1} \mu_{2}\right.} \cdots n_{\left.\mu_{s=1} \mu_{s}\right)} \psi^{(s)},
\end{aligned}
$$


and where $\psi^{*}$ can be substituted by its expression (B1) in terms of an arbitrary \%. Now, it is clear that $\hat{S}^{* \prime \prime \prime}$ vanishes identically, constraining the source $\tau_{\mu} \ldots \mu_{s}$ to be triple traceless too.

As previously pointed out in Ref. 4, gauge invariance of the action imposes the source conservation law

$$
\partial_{\mu} \tau^{* \mu} \tilde{\mu}_{2} \cdots \tilde{u}_{s}=0
$$

where the $\sim$ operator means the (fully symmetric) $\gamma$-traceless part of a given tensor-spinor. For instance, for a rank-s tensor-spinor $\xi_{\mu_{1} \ldots \mu_{s}}(s$ $\geqslant 3$ ),

$$
\tilde{\xi}_{\mu_{1} \cdots \mu_{s}}=\xi_{\mu_{1} \cdots \mu_{s}}-\frac{1}{2}(s+1)\left[\gamma_{\left(\mu_{1}\right.} \xi_{\left.\mu_{2} \cdots \mu_{s}\right)}^{\prime}+\eta_{\left(\mu_{1} \mu_{2} \xi_{\left.\mu_{3} \cdots \mu_{s}\right)}\right.} \xi^{\prime \prime}{ }^{\frac{1}{8}} \gamma_{\left(\mu_{1}\right.} \eta_{\left.\mu_{2} \mu_{3}\right)} \xi_{\mu_{4} \cdots \mu_{s}}^{\prime \prime \prime}\right]+\cdots+a_{s} \eta_{\left(\mu_{1} \mu_{2}\right.} \cdots \eta_{\left.\mu_{s-1} \mu_{s}\right)} \xi^{(s)} .
$$

Finally, let us note that (similarly to what happens in the $\frac{5}{2}$ case) gauge invariance of the action implies the existence of Bianchi identities:

$$
\partial_{\mu} \hat{S}^{* \mu}{ }_{\tilde{\mu}_{2}} \cdots \tilde{\mu}_{3} \equiv 0 \text {. }
$$

When they are decomposed into $3+1$ spatial and temporal components, they determine the constraints contained in the theory:

$$
C_{i_{1} \cdots i_{s}} \sim \hat{S} *^{0} \tilde{i}_{2} \cdots \tilde{i}_{s} \cdot
$$

Taking (B5) into account, we then have, for $s \geqslant 4$ (for $s=3$ the last term vanishes, and for $s=2$ the last two cannot be constructed),

$$
C_{i_{1} \cdots i_{s}} \sim \hat{S}_{i_{2} \cdots i_{s}}^{* 0}-\frac{1}{2(s+1)} \gamma_{\left(i_{2}\right.} \gamma^{\alpha} \hat{S}_{\left.\alpha i_{3} \cdots i_{s}\right)}-\frac{1}{2(s+1)} \delta_{\left(i_{2} i_{3}\right.} \hat{S}_{\left.\alpha \alpha i_{4} \cdots i_{s}\right)}^{* 0}+\cdots
$$

* On leave of absence from Universidad Simon Bolivar, Caracas, Venezuela.

${ }^{1}$ D. Z. Freedman, P. van Nieuwenhuizen, and S. Ferrara, Phys. Rev. D 13, 3214 (1976); S. Deser and B. Zumino, Phys. Lett. $62 \overline{\mathrm{B}}, 335$ (1976).

${ }^{2}$ M. T. Grisaru and H. Pendleton, Phys. Lett. 67B, 323 (1977); see also S. Christensen and M. Duff, Nucl. Phys. B154, 301 (1979).

${ }^{3} \mathrm{~J}$. Schwinger, Particles, Sources, and Fields (Addison-Wesley, Reading, Mass., 1970).

${ }^{4} \mathrm{~J}$. Fang and C. Fronsdal, Phys. Rev. D 18, 3630 (1978).

${ }^{5}$ F. A. Berends, J. W. van Holten, P. van Nieuwenhuizen, and B. de Wit, Phys. Lett. 83B, 188 (1979).

${ }^{6}$ T. Curtright, Phys. Lett. 85B, 219 (1979).
${ }^{7}$ S. Deser, J. H. Kay, and K. S. Stelle, Phys. Rev. D 16 ; 2448 (1977).

${ }^{8} \mathrm{C}$. Aragone and S. Deser, Phys. Lett. 86B, 161 (1979).

${ }^{9} \mathrm{~J}$. Hietarinta, Phys. Rev. D 13, 838 (1976).

${ }^{10}$ B. Konopel'chenko, Fiz. Elem. Chastits At. Yadra

8, 135 (1977) [Sov. J. Part. Nucl. $\underline{8}, 57$ (1977)].

${ }^{11}$ F. A. Berends, J. W. van Holten, P. van Nieuwenhuizen, and B. de Wit, Report No. ITP-SB-79-13, 1979 (unpublished).

${ }^{12} \mathrm{C}$. Aragone and S. Deser, Proceedings of the Supergravity Workshop, edited by P. van Nieuwenhuizen and B. de Wit (North-Holland, Amsterdam, 1979).

${ }^{13}$ C. Aragone and S. Deser, Brandeis report, 1979 (unpublished). 